\title{
TEMA 1-2016: Colgajo Supraclavicular para Recons- trucción Oncológica de Cabeza y Cuello.
}

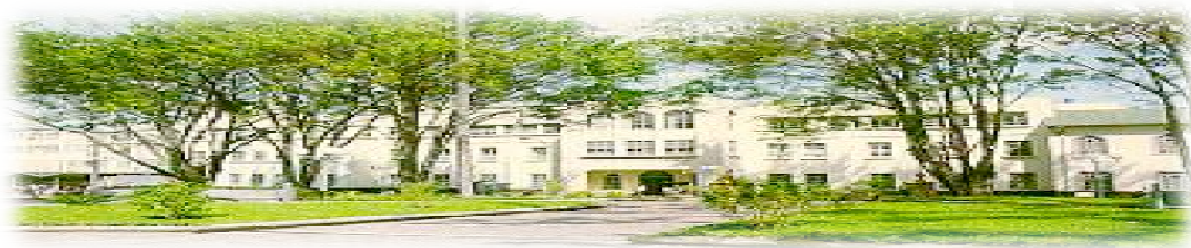

Hospital San Juan de Dios, San José, Costa Rica. Fundado en 1845
ISSN
Recibido:
$22 / 11 / 2015$
Aceptado:
$11 / 12 / 2015$

\author{
Dr. Josías Juantá Castro ${ }^{1}$ \\ Dr. Álvaro Herrera Alfaro ${ }^{2}$
}

\begin{abstract}
${ }^{1}$ Médico Cirujano, Especialista en Cirugía Oncológica, Cirugía de Cabeza y Cuello y Microcirugía, Hospital San Juan de Dios, Caja Costarricense de Seguro Social

${ }^{2}$ Médico Cirujano, Hospital San Juan de Dios, Caja Costarricense de Seguro Social, correo electrónico: aherrera_23@hotmail.com
\end{abstract}

\section{RESUMEN}

A nivel mundial, el cáncer de cabeza y cuello consiste en la sexta malignidad más frecuente. Usualmente el tratamiento quirúrgico requiere de resección amplia, tanto de tejidos blandos como de hueso. Por ende esto implica una reconstrucción que mantenga la estética y funcionalidad para así lograr un buen resultado psicológico para el paciente. ${ }^{(1)}$

El colgajo supraclavicular, debido a su utilidad y versatilidad, puede ser utilizado en los casos de pérdida importante de tejido, esto debido a que posee un amplio arco de rotación que permite alcanzar zonas distantes difíciles a la hora de utilizar otro tipo de colgajo pediculado. Además tiene la ventaja de proporcionar color y textura de piel muy similar al tejido receptor de cabeza y cuello con poca morbilidad del sitio donador. ${ }^{(2)}$

\section{PALABRAS CLAVE}

Cáncer de cabeza y cuello, colgajo supraclavicular, cirugía oncológica, cirugía reconstructiva

\section{ABSTRACT}

The head and neck cancer is the sixth most common malignancy worldwide. The surgery usually requires wide resection of soft tissue and bone. Therefore this implies a reconstruction to maintain the aesthetics and functionality to achieve good psychological outcome for the patient. $^{(1)}$

The supraclavicular flap is very useful and versatile so it can be used in cases of significant loss of tissue, because it has a wide arc of rotation that allows to reach remote areas difficult when 
are use other pedicle flap. It also has the advantage of providing color and texture very similar to skin tissue neck headset and low donor site morbidity. ${ }^{(2)}$

\section{KEY WORDS}

Head and neck cancer, supraclavicular flap, oncologic surgery, reconstructive surgery

\section{DISCUSIÓN}

Se estima que por año se presentan aproximadamente 50 mil casos nuevos de cáncer de cavidad oral, faringe y laringe, lo que representa el $3 \%$ de todos los tumores malignos diagnosticados en Estados Unidos. Y se estima que aproximadamente 11000 pacientes de esos pacientes fallecen debido a dichas enfermedades. ${ }^{(1)}$

El carcinoma de células escamosas es la variante histológica más frecuente representado el $90 \%$ de los casos. Dentro de los factores etiológicos más comunes encontramos: el abuso del alcohol y tabaco, el virus del papiloma humano y la exposición al sol; siendo éste último el más importante para los tumores de piel. ${ }^{(1)}$

En cuanto al manejo de pacientes con tumores de cabeza y cuello, se puede decir que es complejo, esto debido a que se requiere de un equipo multidisciplinario para el manejo, la prevención y el seguimiento de la enfermedad y de las secuelas de cirugía radical, radioterapia y quimioterapia. (1)

Todo paciente que será sometido a una cirugía radical debe ser evaluado por oncólogos quirúrgicos especialistas en cabeza y cuello los cuales deben valorar resecabilidad y estado funcional postoperatorio. La irresecabilidad depende del estadio de la enfermedad, ubicación anatómica y de la experiencia del cirujano así como de la disponibilidad de todo el equipo multidisciplinario. ${ }^{(1)}$

La meta a nivel quirúrgico de los tumores de cabeza y cuello es lograr la resección completa del tumor con la verificación histológica de márgenes libres de tumor: una distancia mayor a $5 \mathrm{~mm}$ entre el tumor y el margen resecado. ${ }^{(1)}$

La reconstrucción del defecto quirúrgico se realiza con técnicas convencionales a discreción del cirujano, pero está técnica no debe interferir en la decisión de realizar resecciones más amplias para obtener márgenes quirúrgicos libres de tumor. ${ }^{(1,3)}$

La resección de tumores del tracto aerodigestivo superior en estadios avanzados usualmente requieren de procedimientos reconstructivos complejos, los cuales son necesarios para mejorar el resultado psicológico, la funcionalidad y estética. $(1,3)$

En este tipo de casos por lo general es necesario utilizar colgajos miocutáneos pediculados regionales o colgajos distantes dado que no es posible el cierre primario debido a la cantidad de tejido resecado, y en muchas ocasiones los tejidos locales están comprometidos por la toxicidad secundaria a la radioterapia o la combinación de la misma con quimioterapia. ${ }^{(1,3)}$

La reconstrucción ideal debería realizarse en un mismo tiempo quirúrgico, sin embargo en algunas ocasiones se debe confirmar histológicamente los márgenes de resección por lo que se debe postergar la reconstrucción para un segundo tiempo quirúrgico. ${ }^{(1,3)}$

El tipo de reconstrucción va a depender del sitio anatómico, extensión de la resección y aún más $i_{(4)}$ portante, la expectativa de la vida del paciente.

Existen múltiples técnicas para la reconstrucción luego de las resecciones amplias de tumores de cabeza y cuello, las cuales incluyen cierre por segunda intención, cierre primario, injertos de piel y los colgajos. ${ }^{(3)}$

Los colgajos pueden incluir colgajos locales o pediculados distantes y colgajos libres. Se prefiere la utilización de tejidos propios del paciente ya que tiene la ventaja de evitar el rechazo del tejido donador y la necesidad de utilizar la inmunosupresión por largo tiempo. La combinación de las diferentes técnicas más la utilización de prótesis aloplásticas permiten una gran gama de opciones para la reconstrucción lo cual puede tener mejor resultado que utilizar una única técnica. ${ }^{3,4)}$

Para obtener un resultado satisfactorio funcional y estético el color y la textura del colgajo son siempre esenciales. Sin embargo, el defecto del sitio donador se debe reducir al máximo, evitando la discapacidad funcional y estética del mismo. ${ }^{(3,4)}$

\section{Colgajo supraclavicular}

Historia 
En 1920 Gillies describe el concepto básico de que entre más cercano esté el sitio donador del sitio receptor habrá mayor similitud en color y textura de la piel. ${ }^{(5)}$

El colgajo originado de la red vascular de la arteria cervical y de su rama, la arteria supraclavicular tiene una larga historia de controversia. En 1842 Mutter fue el primero en describir un colgajo de la región supraclavicular extendiéndose hasta el hombro. Sin embargo, luego de varias modificaciones y mayor valoración de la vasculatura, en 1949 Kazanjian y Converse lo describen en su publicación: el tratamiento quirúrgico de las lesiones faciales como "en charretera" en alusión a la divisa militar utilizada en el hombro del uniforme; o como flap acromial. ${ }^{(5,6)}$

Actualmente el colgajo supraclavicular representa una evolución de otros colgajos, como el colgajo descrito por Demergasso en 1979; el cual fue definido por Mathes y Nahai como una extensión faciocutánea del colgajo lateral superior del trapecio en la región lateral del brazo. ${ }^{(5,6)}$

Lamberty y Cormack, en 1983, definen el colgajo como el colgajo cervicohumeral extendido y describieron la anatomía vascular del hombro y la axila. ${ }^{5,6)}$

En 1997 Pallua publicó una modificación del colgajo y lo define como el colgajo en isla de la arteria supraclavicular. En el año 2000 el mismo Pallua describe la vascularidad exacta y la posibilidad de tunelización para mejorar la movilidad del colgajo y para reducir la cicatrización de la zona donadora. ${ }^{(5,6,7)}$

\section{Anatomía}

El colgajo supraclavicular está basado en la arteria cervical transversa y su rama la arteria supraclavicular. Estudios cadavéricos demostraron que la arteria supraclavicular surge aproximadamente entre 3 a $5 \mathrm{~cm}$ del origen de la arteria cervical transversa, en un triángulo formado por el tercio medio de la clavícula, el músculo esternocleidomastoideo y la vena yugular externa. Rara vez, la arteria supraclavicular puede surgir de la arteria supraescapular. ${ }^{(5,8)}$

La arteria cervical transversa se ubica 3 a $4.5 \mathrm{~cm}$ por encima de la articulación esternoclavicular (figura 1), y el origen de la arteria supraclavicular se encuentra a $6-8.5 \mathrm{~cm}$ de ésta articulación. La distancia entre el origen de la arteria supraclavicular y el punto en que penetra a la fascia profunda varía de 2 a $4.5 \mathrm{~cm}$. El diámetro es típicamente de 1.1 a $1.5 \mathrm{~mm} .^{(5,6,7,8)}$

Figura 1 Anatomía arteria cervical transversa

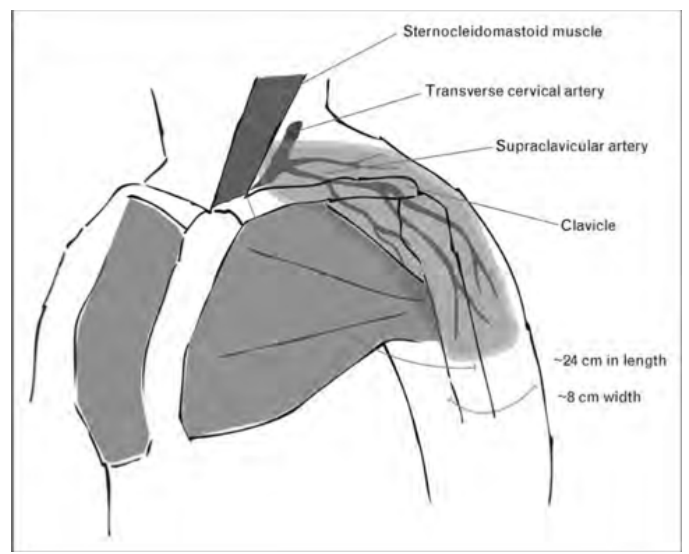

Estudios recientes en cadáveres frescos utilizando angiografía tomográfica han confirmado que la perfusión del colgajo está basado en la arteria supraclavicular y la parte más distal del colgajo recibe flujo sanguíneo de vasos interperforantes y de plexos subdérmicos. ${ }^{(5,6,7,8)}$

En todos los cadáveres estudiados se encontraron dos venas que drenan el colgajo (figura 2). Una vena recorre el colgajo adyacente a la arteria y drena en la vena cervical transversa y tiene un diámetro aproximado de $0.22 \mathrm{~cm}$. La segunda vena es tributaria de la vena yugular externa y tiene un diámetro medio de $0.24 \mathrm{~cm}$; en algunos casos ésta última vena puede drenar en la vena subclavia directamente. ${ }^{(5,6,7,8)}$

Figura 2 Anatomía vena supraclavicular

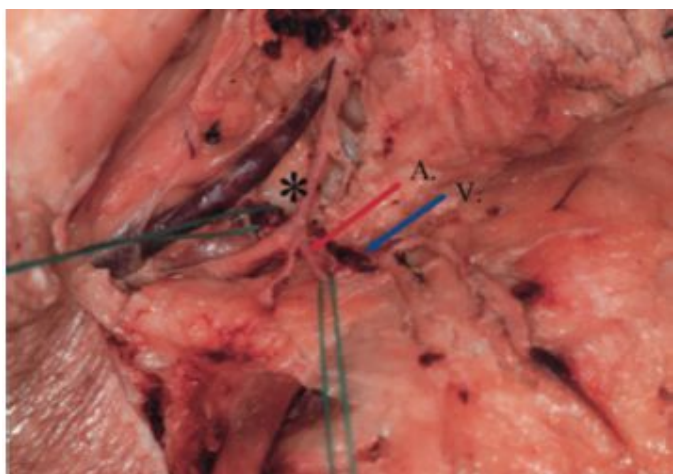


Los estudios realizados de perfusión del colgajo sugieren que las dimensiones máximas de colgajo que se pueden obtener son de hasta $35 \mathrm{~cm}$ de longitud y $12 \mathrm{~cm}$ de ancho.(figura 3$)^{(5,7)}$

Figura 3 Longitud de colgajo supraclavicular

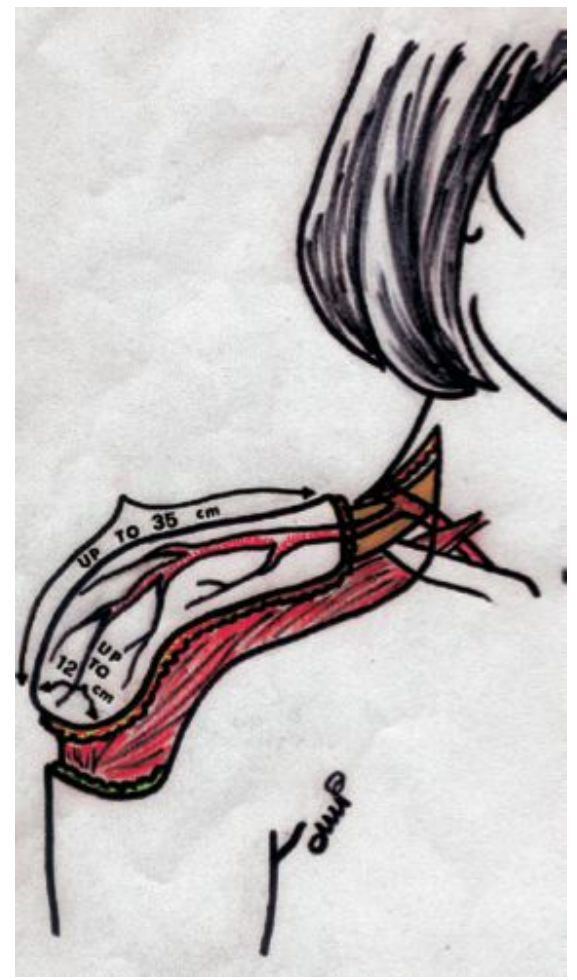

La inervación del colgajo está dada por un grupo de tres a cuatro ramas nerviosas principales arborizadas que se encuentran en la fosa supraclavicular provenientes del cuello y el hombro originadas del tercer y el cuarto nervio cervical. En la mayoría de los casos se puede encontrar una o dos ramas provenientes del plexo cervical los cuales recorren la región del hombro al lado de los vasos sanguíneos. (figura 4) ${ }^{(9)}$

Figura 4 Inervación colgajo supraclavicular

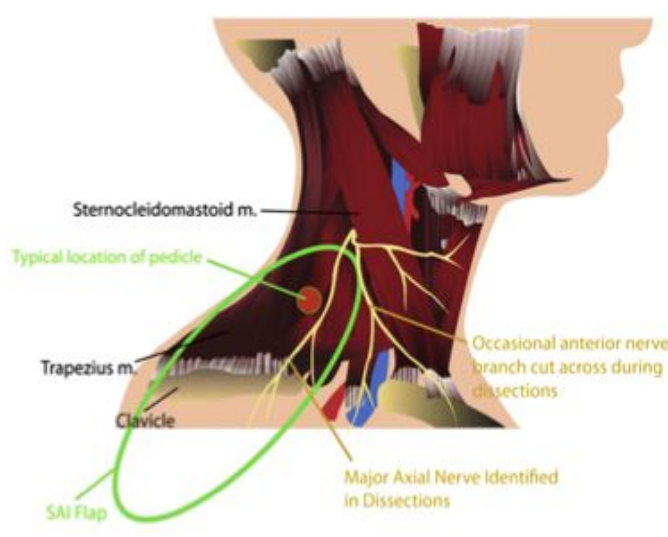

Técnica quirúrgica

La arteria y la vena supraclavicular deben ser reconocidas preoperatoriamente usando una sonda de ultrasonido doppler. Posteriormente se esboza el pedículo del colgajo.(figura 5) ${ }^{(5,6,7,10)}$

\section{Figura 5 Esbozo del colgajo}

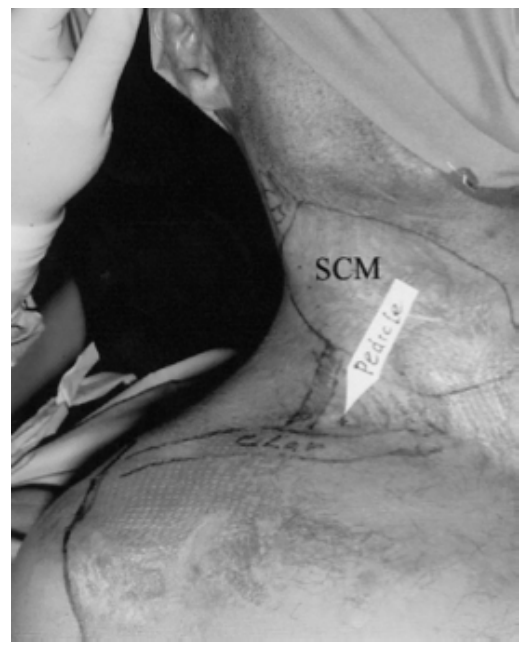

Se realiza resección del tumor y confirmación histológica de los márgenes quirúrgicos del mismo, de no ser posible confirmar los márgenes de resección adecuados es mejor realizar una reconstrucción en una segunda intervención. $(5,6,7,10)$

Durante la elevación del colgajo, el paciente se coloca en decúbito dorsal, con un ángulo de 45 grados. La incisión se realiza desde el sitio donde se marcó el pedículo hasta llegar al músculo deltoides. El colgajo se diseca en un plano subfacial de lateral hasta medial. (Figura 6) ${ }^{(5,6,7,10)}$ 
Los vasos perforantes provenientes del músculo deltoides deber ser seccionados. En el tercio medio del colgajo, el pedículo puede ser identificado fácilmente por transiluminación. El colgajo es delgado lo que asemeja el color y la textura de la región baja de la cara, el cuello y la zona superior del tórax. $(5,6,7,8)$

\section{Figura 6 Levantamiento del colgajo}

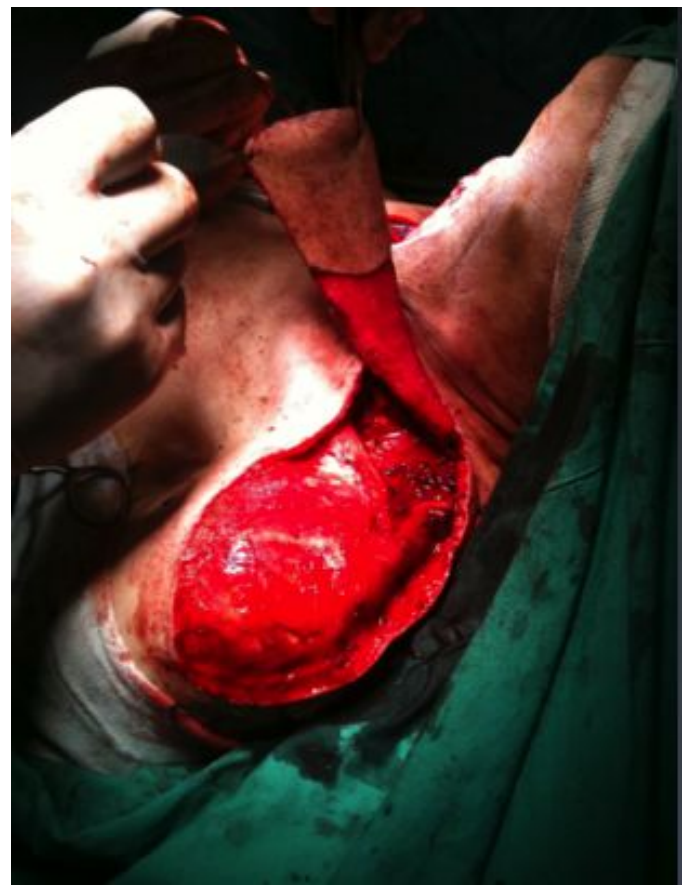

Al alcanzar el límite medial de la insición del colgajo, en el sitio del origen del pedículo, se incide la piel superficialmente teniendo mucha precaución de no lesionar los vasos supraclaviculares. Posteriormente se prepara el pedículo a nivel subcutáneo, en la región superior y a nivel subfacial en el sitio inferior del pedículo. Una vez disecado el pedículo se realiza la tunelización del colgajo para llevarlo al sitio receptor. $(5,6,7,8)$

El sitio donador puede ser cerrado primariamente con múltiples suturas subcutáneas para aproximar los bordes de la herida (figura 7). Aún en colgajos tan amplios como $16 \mathrm{~cm}$ no es necesario utilizar injertos de piel para cerrar el defecto. $(5,6,7,8,10)$

Figura 7 Cierre de sitio donador

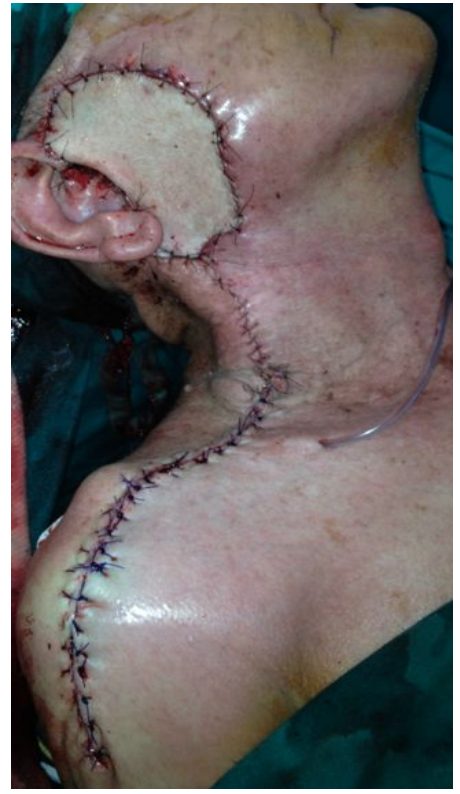

El colgajo supraclavicular posee un eje axial grande lo cual permite ser utilizado para cubrir defectos de casi la totalidad del cuello. Sin embargo no es lo suficientemente grande como para cubrir defectos de toda la hemicara. Por tal razón se ha descrito una técnica quirúrgica en 2 tiempos, en la cual en el primer tiempo quirúrgico se levanta el colgajo, se coloca un expansor por debajo del mismo y se realiza un retardo de colgajo. En un segundo tiempo quirúrgico se puede utilizar el colgajo pre-expandido el cual va a resultar en un colgajo de mayor tamaño para poder reconstruir un defecto mayor. ${ }^{(11)}$

\section{Figura 8 Expansor del colgajo supraclavicular}

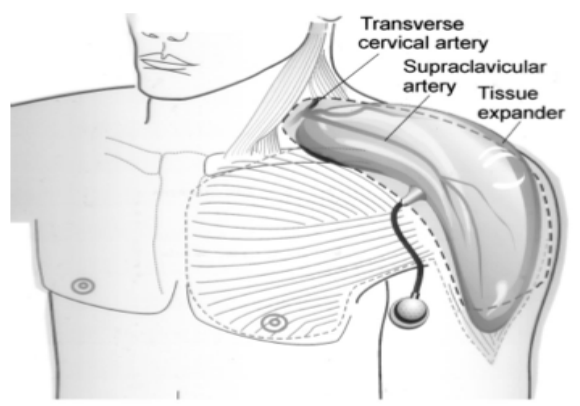

Aplicaciones del colgajo en cirugía de cabeza y cuello.

El colgajo en isla de la arteria supraclavicular se ha utilizado principalmente para la reconstruc- 
ción de defectos cutáneos provocados por quemaduras. Sin embargo, más recientemente se está utilizando para la reconstrucción luego de la resección oncológica de tercio inferior de cara y la totalidad de cuello, por ejemplo reconstrucción de faringe, traqueostomías, restauración de piso de boca y cierre de defectos cutáneos. ${ }^{(2,5,10,12)}$ Este colgajo se ha utilizado con éxito en pacientes con múltiples comorbilidades incluyendo obesidad, desnutrición, diabetes e historia de fumado. La única contraindicación para su utilización es el antecedente de disección bilateral de cuello y de irradiación de cuello. ${ }^{(2,5,12)}$

\section{Ventajas y desventajas}

El colgajo supraclavicular es delgado, flexible, vascularizado, es regional y de base axial con una pigmentación muy similar a la piel de cabeza y cuello. También es un colgajo fasciocutáneo con mínima morbilidad del sitio donador de hombro sin provocar disfunción de la movilidad de miembro superior. Una desventaja es que por su tamaño está limitado para la reconstrucción de defectos de tercio inferior de cara, lo cual se podría solucionar con la técnica de dos tiempos utilizando un expansor, descrita anteriormente. $(2,5,11,12)$

\section{Experiencia Hospital San Juan de Dios}

El Hospital San Juan de Dios cuenta con una experiencia de más de diez pacientes que han sido sometidos a cirugía de colgajo supraclavicular. Los pacientes operados contaban ,a la hora de ser sometidos a la cirugía, con una edad promedio de 66 años y presentaban como principal diagnóstico: defecto de piso de boca, defecto de lecho parotídeo y cuello, fibrosarcoma de mandíbula, defecto de órbita ocular, defecto de pabellón auricular, fístula faringocutánea, exposición de material postreconstrucción de mandibula, entre otros. Siendo el carcinoma epidermoide el estirpe histológico más frecuente dentro de los pacientes operados. Los colgajos supraclaviculares contaban con medidas promedio de $19.3 \mathrm{~cm}$ de largo y $7,1 \mathrm{~cm}$ de ancho. La estancia postoperatoria promedio fue de 18 días y la complicación postquirúrgica más frecuente fue sepsis de herida quirúrgica, la cual resolvió satisfactoriamente en todos los casos con antibioticoterapia.
Cabe destacar que un paciente debido a la extensión de sus lesiones requirió reconstrucción con doble colgajo supraclavicular, el cual evoluciono de manera satisfactoria.

\section{CONCLUSIÓN}

El colgajo supraclavicular constituye una buena alternativa para la reconstrucción locorregional de cabeza y cuello con mínima morbilidad del sitio donador. Este colgajo es una excelente opción para pacientes que no son candidatos para cirugía microvascular, pacientes con alto riesgo de recurrencia de la enfermedad y patología oncológica avanzada.

En este trabajo se demuestran las diferentes utilidades que puede tener el colgajo supraclavicular para la reconstrucción de defectos luego de la cirugía oncológica de cabeza y cuello, obteniendo muy buenos resultados estéticos y funcionales. Además hubo pocas complicaciones asociados al uso de este colgajo.

Al ser un colgajo fácil de obtener y permitir gran variedad de reconstrucciones con mínima morbilidad del sitio donador, este colgajo debe estar presente en el armamento quirúrgico de cirujanos de cabeza y cuello.

\section{BIBLIOGRAFÍA}

1. National comprehensive cancer network. Clinical practice guidelines in oncology, head and neck cancers. Version 2.2013.

2. Sandu K Monnier P. Supraclavicular flap in head and neck reconstruction: experince in 50 consecutive patients. European archives of otorhinolaryngology. 2012; 269 (4): 1261-7.

3. Mitchell O. Current advances in facial reconstructive surgery following head and neck cancer surgery. Plastic surgical nursing. 2012; 32 (1): 6-9. 
4. Wehage I Fansa H. Complex reconstructions in head and neck cancer surgery: decision making. Head and neck oncology. 2011; 3 (14): $2-11$

5. Pallua N Magnus E. The tunneled supraclavicular island flap: An optimized technique for head and neck reconstruction. Plastic and reconstructive surgery. 2000; 105 (3): 842-51.

6. Kim R Izzard M Patel R. Supraclavicular artery island for reconstructing defects in the head and neck region. Current opinion in otolaryngology and head and neck surgery. 2011, 19: $248-250$.

7. Di Benedetto $G$ Aquinati A Pierangeli $M$ Scalise A Bertani A. From the charretera to the supraclavicular fascial island flap: Revisitation and further evolution of a controversial flap. Plastic and reconstructive surgery. 2005; 115 (1): 70-76.

8. Cadena-Piñeros E. Colgajo fasciocutáneo en isla de la arteria supraclavicular: alternativa segura en la reconstrucción de la región preauricular. Revista colombiana de cancerología. $2010 ; 14(2)$ : 116-20.

9. Sands T Martin J Simms E Henderson M Friedlander P Chiu E. Supraclavicular artery island innervation: anatomical studies and clinical implications. Journal of plastic, reconstructive and aesthetic surgery. 2012 65, $68-71$.

10. Anand A Tran E Hasney C Friedlander P Chiu E. Oropharyngeal recostruction using the supraclavicular artery island flap: A new flap alternative. Plastic and reconstructive surgery. 2012; 129 (2): 438-41.
11. Pallua N von Heimburg D. Pre-expanded ultrathin supraclavicular flaps for full face reconstruction with reduced donor-site morbidity and without the need for microsurgery. Plastic and reconstructive surgery. 2005; 115 (7): 1837-44.

12. Chiu E Lui P Friedlander P. Supraclavicular artery island flap for head and neck oncologic reconstruction: Indications, complications and outcomes. Plastic and reconstructive surgery. 2009; 124 (1): 115-23.

\section{CONFLICTO DE INTERÉS Y/O AGRADE- CIMIENTOS}

Los autores declaran que no existió ningún conflicto de interés en el presente reporte. 